\section{The power of individual cultural values in global virtual teams}

\author{
Audra I. Mockaitis
}

Monash University, Australia

Elizabeth L. Rose

Aalto University School of Economics, Finland

\section{Peter Zettinig}

University of Turku, Finland
International Journal of Cross Cultural Management 12(2) 193-210

(c) The Author(s) 2012 Reprints and permissions: sagepub.co.uk/journalsPermissions.nav DOI: 10.1 I 77//4705958I2439868 ccm.sagepub.com

@SAGE

\begin{abstract}
This paper investigates the perceptions of members of 43 culturally diverse global virtual teams, with respect to team processes and outcomes. Despite widespread acknowledgement of the challenges presented by cultural differences in the context of global teams, little is known about the effect of these differences on team dynamics in the absence of face-to-face interaction. Using a student-based sample, we study the relationship between global virtual team members' individualistic and collectivistic orientations and their evaluations of trust, interdependence, communication and information sharing, and conflict during the team task. Our results suggest that a collectivist orientation is associated with more favorable impressions regarding global virtual team processes and that cultural differences are not concealed by virtual means of communication.
\end{abstract}

\title{
Keywords
}

Cultural values, global teams, individualism/collectivism, virtual teams

Multicultural teams face a myriad of challenges not faced by single-culture teams. Cultural differences within teams have the potential to pose barriers with respect to communication, relationship building, cooperation, and trust, influencing team members' views of the group and both their own and others' participation in it. Greater cultural diversity within the team is expected to result in more pronounced differences in attitudes and behaviors.

\section{Corresponding author:}

Audra I. Mockaitis, Department of Management, Monash University, Australia.

Email: audra.mockaitis@monash.edu 
Technological advances of the last decade have generated expanded use of virtual teams by a variety of organizations; however, there has been little research to date on multicultural virtual teams. Jarvenpaa and Leidner (1999) considered global virtual teams, defined as geographically and culturally diverse groups that are temporary, have minimal face-to-face contact, communicate through electronic media, and whose members work across temporal and spatial boundaries to coordinate their activities toward the attainment of common goals. In addition to the cultural barriers that are encountered in traditional global teams, global virtual teams face the challenges of working across time and space, without the ability to observe the behaviors of other team members during communication.

Our premise is that team members' cultural attributes matter in the context of global virtual teams. The extant literature suggests that differences with respect to individualism and collectivism (I/C) are particularly important to attitudes toward teamwork; thus, our focus in this research is on this aspect of culture. While there is much evidence to suggest that collectivists are especially accepting of teamwork and willing to forego personal interests for the sake of group goals, it is not clear how a person's position on the I/C continuum is related to his or her attitudes toward global virtual teams. Are I/C distinctions as pronounced, and does the $\mathrm{I} / \mathrm{C}$ dimension have as strong a relationship with teamwork, when teams are short-lived and operate without face-to-face interaction? The ad hoc nature of most global virtual teams means that team members have very limited time and opportunity to form interpersonal relationships and resolve in-group/out-group distinctions. Understanding more about the relationship between the $\mathrm{I} / \mathrm{C}$ dimension and perceptions regarding global virtual teams will help to shed light on the question of whether people behave in prototypically individualistic or collectivistic ways, regardless of the context of their interaction.

People with strong collectivist orientations typically seek group goals through the development and maintenance of relationships within the group, while individualists tend to be more taskoriented (Gomez et al., 2000). This suggests that collectivists may be more comfortable in situations in which the outcome depends on collective effort. However, developing trust, relationships, and loyalty to groups takes time, which is often quite limited in the context of global virtual teams. When faced with similar challenges and an inability to communicate face to face, will individualists and collectivists have different post hoc perspectives about working in multicultural teams? Specifically, will collectivists have more positive evaluations of work in virtual teams, or will the conditions of global virtual teams favor individualists?

In this paper, we test the applicability of the theoretical assumptions regarding the $\mathrm{I} / \mathrm{C}$ dimension and teamwork to a culturally diverse global virtual team setting. Using a sample of student participants, we assess the relationship between team members' self-assessments of their own positions on the $\mathrm{I} / \mathrm{C}$ continuum and their evaluations of the degrees of trust, cooperation, and conflict in teams, following the completion of a global virtual project. We begin with an overview of the literature on multicultural teams and the development of hypotheses about the $\mathrm{I} / \mathrm{C}$ dimension in the context of global virtual teams. This is followed by descriptions of the setting for our empirical study and our research methods, then a description of our results. The paper concludes with findings, implications of the study, and suggestions for future research.

\section{Background}

\section{Some challenges of virtual teams in a multicultural setting}

The development of virtual teams has been facilitated by rapid advances in information and communication technologies. This new type of work group is differentiated from traditional teams by 
permeable interfaces and boundaries (Jarvenpaa and Leidner, 1999). Characteristics such as rapid formation and the ease of reorganization and dissolution give virtual teams advantages with respect to meeting the quickly changing requirements of the highly dynamic international marketplace (e.g. Kristof et al., 1995; Mowshowitz, 1997).

However, virtual teams are not without challenges, many created by the lack of face-to-face contact among members. Handy (1995: 46) questioned the effectiveness of virtual teams, arguing that 'trust needs touch'. Jarvenpaa and Leidner (1999), among others, suggested that collocation, missing in virtual teams, increases social similarity and values, and shared expectations, improving team functionality. Dysfunctions such as low individual commitment, role overload, role ambiguity, absenteeism, and social loafing are raised as potential problems with virtual teams (O'Hara-Devereaux and Johansen, 1994). Nohria and Eccles (1992: 304-5) adopted a strong stand, stating that 'you cannot build network organizations on electronic networks alone ... If so, ... we will need an entirely new sociology of organizations.' Jarvenpaa and Leidner (1999) noted that virtual teams require the development of a shared social context, in order to build essential trust.

In the context of an organization that operates internationally, virtual teams are subject to the additional complexity brought about by interactions among culturally diverse team members. While multicultural communications are more complicated, there are also indications that culturally diverse and geographically dispersed virtual teams, operating via information and telecommunication networks, offer some considerable benefits. One such positive is that the greater variation of perspectives among members of multicultural virtual teams offers more potential resource combinations, allowing for alternative approaches and innovative problem-solving. While substantial research has focused on cross-cultural communication (e.g. Matveev and Nelson, 2004) and on teamwork via information and communication technology (e.g. Jarvenpaa and Leidner, 1999), there is still a great deal to be developed with respect to understanding interactions of cross-cultural behavior in global virtual teams.

\section{The individualism-collectivism dimension in diverse groups}

The individualism-collectivism (I/C) dimension is widely viewed as a particularly important aspect of culture (Hofstede, 1984; Maznevski et al., 2002; Triandis, 2004) and with respect to individual behavior in the context of groups and teams. Hofstede (2001: 209) defined individualism as 'the relationship between the individual and the collectivity that prevails in a given society'. Whereas individualists tend to identify with the self, collectivists tend to identify with the group to which they belong and give priority to group goals and rewards. In individualist societies, ties between individuals are loose, while in collectivist societies people are integrated into tightly knit ingroups that protect the individual in exchange for loyalty (Hofstede, 2001). Collectivists are more motivated by the norms of and obligations to the group, and tend to give priority to group over personal needs. Individualists, on the other hand, are more motivated by personal goals and are likely to prioritize personal needs; their involvement with others tends to be more calculated, rather than based on duty or obligations (Triandis, 1995). Collectivists are also more likely to attribute individual behavior to external factors, while individualists attribute behavior to personality and other internal aspects (Triandis, 2004).

While Hofstede's (1984) individualism dimension, at the culture level, suggests that individualism and collectivism are distinct poles of a single dimension, research at the level of the individual has shown that both individualist and collectivist aspects are present in all cultures and pronounced to different degrees (Maznevski et al., 2002; Triandis, 1994). Although respondents from collectivist cultures tend to score higher on collectivism measures at the individual level, and vice versa 
(e.g. Bierbrauer et al., 1994), there is evidence of considerable variation within cultures. People may behave in ways more typical of individualists or collectivists, depending on the situation. This has been illustrated in studies indicating that, even in highly collectivist cultures, social loafing still occurs in group environments when, for example, collectivists are confronted with out-group situations or when they exhibit individualist tendencies (e.g. Earley, 1989). Thus, while studying the I/C dimension at the culture level is useful for comparing general tendencies across cultures, some important characteristics may be ignored if we reduce each nationality to a single score on an I/C scale (Kim et al., 1990; Maznevski et al., 2002).

At the personal level, the terms 'idiocentrism' and 'allocentrism' have been used to describe individualists and collectivists, in order to avoid conceptual confusion (e.g. Kagitcibasi, 1994; Triandis et al., 1985). For this micro level, the I/C dimension has been broken down into different factors, not necessarily on opposing extremes (Triandis, 2004), so that people are seen to exhibit both individualist and collectivist behaviors to varying degrees. Triandis (1995) identified different types of individualists and collectivists: vertical and horizontal. The horizontal I/C dimension describes people who stress equality, while the vertical dimension reflects an acceptance of hierarchy. Consideration of these more subtle distinctions in the $\mathrm{I} / \mathrm{C}$ measure allows for an explanation of how cultures that rate similarly with respect to macro-level I/C, such as Sweden and the USA, are, in fact, so different. Whereas Americans tend to value independence, self-reliance, and individuality, Swedes are more likely to value self-reliance while being uncomfortable with standing out from the crowd (Triandis, 1995).

Reviewing 180 studies on cultural values, spanning the two decades since Hofstede's seminal work, Kirkman et al. (2006) identified a tendency by researchers to confuse individual- and culturelevel measures. Our study seeks to bridge this gap in the literature, by incorporating a consideration of values at both the micro and macro levels. In this way, we take into account the notions that some societies may be more or less individualistic, on average, than others, while people's cultural values are likely to vary within countries.

In this work, our focus is primarily on the individual, and we develop hypotheses regarding the relationship between the $\mathrm{I} / \mathrm{C}$ dimension at the personal level and attitudes toward work in multicultural teams. In this way, we allow for variation between a particular individual and the general orientation of his or her national culture. Avoiding the widespread mistake of assigning countrylevel scores to individuals (Hofstede, 1984, 2001; Kirkman et al., 2006), we incorporate a consideration of both the personal-level collectivism measure of Yamaguchi (1994) and the individualism index scores of Hofstede (1984, 2001).

Past research suggests that the I/C dimension is associated with cooperative behavior in groups, group performance, and evaluations (Cox et al., 1991; Gomez et al., 2000; Wagner, 1995). Triandis (2004) noted that collectivists tend to value organizational commitment and loyalty. In a study of 15 subsidiaries of a US multinational corporation, Palich et al. (1995) observed a negative relationship between individualism and employee commitment. Kirkman and Shapiro (2001) identified a positive association between collectivism and commitment. Gomez et al. (2000) noted that collectivists, more than individualists, value harmony and cooperation in the workplace.

Trust is a critical factor for the effective functioning of groups (Mayer et al., 1995). Reviewing interpersonal measures of trust, Lewicki et al. (2006) noted that some studies have shown that little prior contact is associated with high levels of interpersonal trust at the onset (e.g. Kramer, 1994) and that willingness to trust depends on societal culture (e.g. Fukuyama, 1995). However, the literature shows inconsistent results with respect to I/C and trust in groups and teams. Jarvenpaa and Leidner (1999) argued that, because individualists are less influenced by group membership and are more 
open in communicating, compared with collectivists, they should demonstrate more trusting behavior; since collectivists rely more on stable relationships, which take longer to build, trust should, in turn, take longer to develop. However, Jarvenpaa and Leidner (1999) did not find significant differences between individualists and collectivists, in terms of willingness to trust other members in global virtual teams. Kiffin-Petersen and Cordery (2003) argued for a negative relationship between individualism and both trust and preference for teamwork. Kirkman and Shapiro $(1997,2001)$ also found that individualists are more resistant to teams and interdependence. Collectivists are viewed as valuing loyalty to the in-group and the interdependence of group members. In contrast, group membership for individualists is presumed to be largely calculative (Hofstede, 1984); relationships are likely to be more superficial and short-term, and individualists in groups are expected to act in their own self-interests. Such tendencies may be even more pronounced in temporary groups, and when the lack of a shared context decreases the potential for developing trust (Jarvenpaa and Leidner, 1999).

The expected lower commitment to groups by individualists should be less conducive to trust. However, collectivists in global virtual teams arguably face similar barriers, induced by the transient nature of the groups. Still, we expect that, despite the inherent challenges of global virtual teams (especially the short lifespan that inhibits the development of relationships), collectivists will tend to have more positive views of the level of trust present in the team, based on the collectivist orientation toward loyalty and acceptance of interdependence (Kiffin-Petersen and Cordery, 2003). This leads to our first hypothesis:

Hypothesis 1: There will be a positive relationship between a person's collectivist orientation and his or her evaluation of trust in the multicultural global virtual team.

In addition to trust, cooperation and interdependence are essential for the coordination of work in a team environment (Wagner, 1995). A key characteristic of teams is that members must depend on one another and pool their resources to attain common team goals (Mayer et al., 1995). Because teams are, by nature, interdependent, members must be willing to cooperate with one another (Kiffin-Petersen and Cordery, 2003). Members from individualist and collectivist cultures are likely to have dissimilar perceptions regarding the extent of interdependence among team members and differ in their willingness to engage in cooperative behavior because of their divergent understandings of the factors central to goal attainment. The literature suggests that individualists tend to value task accomplishment and task inputs in the context of teamwork, while collectivists are more likely to focus on the maintenance of relationships, with the goal of group cohesiveness and success (e.g. Gomez et al., 2000; Hofstede, 1984; Kim et al., 1990). As a result, managers in individualist cultures are generally more focused on performance, while managers in collectivist cultures express more concern for relationships (Triandis, 2004).

We expect that these tendencies will also be observable at the personal level. Thus, people who are strongly individualist should be more likely to prefer dividing group tasks, allocating them to team members, and working relatively independently. In contrast, those who are strongly collectivist are apt to be motivated by tasks that require working together to achieve a common outcome (Sosik and Jung, 2002). Because the very nature of teamwork implies a collective outcome, collectivists, with higher reliance on others and stronger expectations for sharing tasks and information, should exhibit stronger perceptions of interdependence and cooperation as means to accomplishing group tasks. We expect this distinction to be heightened in the context of virtual teams, given the lack of face-to-face contact and relative ease of task compartmentalization. Thus, we thus hypothesize that: 
Hypothesis 2: There will be a positive relationship between a person's collectivist orientation and his or her perceptions of the interdependency among members of the multicultural global virtual team.

Hypothesis 3: There will be a positive relationship between a person's collectivist orientation and his or her perceptions of the dependence on communication and/or information sharing to complete tasks within the multicultural global virtual team.

The notion of conflict - how it is perceived and managed - is also critical to the functioning of teams. Triandis (1995) suggested that collectivists have a threshold for conflict that far exceeds that of individualists, who are less patient and tend to focus on the content (as opposed to context) of communication. Jehn (1995) identified and measured two types of conflict in groups: relationship and task. Relationship conflict is a result of interpersonal tension in the group, and leads to anxiety and emotional reactions. Task conflict is a disagreement over the task at hand.

In highly interdependent groups, relationship conflict is expected to have a stronger negative effect on outcomes. In contrast, task conflict has the potential for positive effects, such as when it is in the form of constructive criticism or the exchange of information and ideas (Jehn, 1995). In the context of short-duration virtual teams, task conflict should be more salient than relationship conflict. In addition to pressure to arrive at a common solution in a short time, the lack of face-to-face contact and short lifespan may serve to inhibit the development of strong relationships. Because individualists tend to place more emphasis on task accomplishment (Gomez et al., 2000), we expect them to find that conflict related to the task at hand, rather than to relationships, is more focal. In contrast, collectivists are likely to have more neutral evaluations of conflict in the team, regardless of whether the conflict is related to task or relationship. This leads to our final hypotheses:

Hypothesis 4: There will be a negative relationship between a person's collectivist orientation and his or her perceptions about the level of relationship conflict among members of the multicultural global virtual team.

Hypothesis 5: There will be a negative relationship between a person's collectivist orientation and his or her perceptions about the level of task conflict among members of the multicultural global virtual team.

\section{Methods}

\section{Sample and data collection}

The data for this study were collected from participants in a three-week-long project, in which business plans were developed by global virtual teams. Each team was comprised of up to 10 participants, generally including representatives of at least four countries and, when possible, different continents, to ensure that the teams were truly global. Approximately 20 percent of the participants were undergraduate students at a New Zealand university, for whom the project constituted a course requirement; other team members participated on a volunteer basis and were largely, but not exclusively, university students. The project was initiated as part of an undergraduate course in New Zealand. A website was developed, and participants were recruited by advertising the project at universities throughout the world. Participants included undergraduate and graduate students, along with working adults. Because of team members' geographic dispersion, no faceto-face contact was possible during the course of the project, and teams were reliant on virtual means of communication. 
Table I. Sample breakdown by nationality

\begin{tabular}{lrr}
\hline Country/ldentity & $n$ & $\%$ \\
\hline New Zealand & 27 & 25.7 \\
China & 24 & 22.9 \\
Finland & 6 & 5.7 \\
Germany & 6 & 5.7 \\
Lithuania & 5 & 4.8 \\
Malaysia & 4 & 3.8 \\
United States & 4 & 3.8 \\
India & 3 & 2.9 \\
Macau & 3 & 2.9 \\
Rwanda & 3 & 2.9 \\
Bulgaria & 2 & 1.9 \\
Canada & 2 & 1.9 \\
Uganda & 2 & 1.9 \\
I each from: Chile, Iran, Ireland, Japan, Kenya, & 14 & 13.3 \\
Nigeria, Philippines, Poland, Singapore, South Korea, & & \\
Switzerland, Thailand, Turkey, Vietnam & & 100 \\
Total & 105 & \\
\hline
\end{tabular}

A questionnaire was distributed to all participants upon completion of the project, to assess their perceptions of their teams' dynamics and processes. The survey was administered electronically (by email) to all team members. Although 351 people had initially registered for the project, for a total of 48 teams with representation from 30 countries, not all of those registered actually participated in the team process. Some teams were unable to contact registered members throughout the duration of the project, but included these members in their final indication of team size. Questionnaires were sent to all team members, regardless of the extent of their participation in the project. This situation makes calculation of an effective response rate rather complicated. We received 105 responses from 43 teams, which had a total of 312 active members. Thus, our effective response rate is approximately 30 percent.

Our sample has a relatively even gender split (45.6 percent male and 54.3 percent female), and 82.6 percent of the respondents are either enrolled in university or hold a university degree. While most of the respondents are students residing in New Zealand (67.6 percent), the sample includes respondents from 32 countries, representing 27 nationalities. Because national identity is an increasingly complex construct, we employ several measures to gauge respondents' nationalities, including birth country, the extent to which they identify with their country of birth, and the country with which they most identify. Where at least two of these responses were concurrent, that country was input as the respondent's nationality; otherwise, birth country was recorded as the respondent's nationality. Table 1 shows the breakdown of the sample by identified nationality. The difference between the numbers of countries (32) and nationalities (27) arises from the fact that the country in which a person currently lives is not necessarily the best measure of his or her national identity. Many participants in this study are either international students or foreign nationals working abroad. Some respondents also report identifying with a culture other than the dominant one of their country of birth and/or residence. 


\section{Research instrument}

The survey instrument was developed to enable measurement of the respondents' personal I/C orientations, and to elicit their perceptions of the teamwork process during the project. The questionnaire was developed and distributed in English. Despite the large number of nationalities represented in the study, the working language for all of the teams in the project was English.

\section{Measures}

Dependent variables. We employ five dependent variables for this study, each measuring respondents' perceptions of a specific aspect of their team processes. The multi-item constructs are developed as the means of the responses to the applicable items, each based on seven-point Likert scales $(1=$ strongly disagree and $7=$ strongly agree $)$ and selected based on theory and iterative reliability assessments.

The first dependent variable, Trust, is based on Erdem and Ozen (2000). Items in this construct pertain to respondent opinions about the qualifications of team members, the extent to which members shared information and resources, trust in the expertise of team members, being able to count on help from team members, the level of respect within the team, and the extent to which ideas and suggestions were encouraged during team interactions. Higher scores indicate stronger perceived trust within the team. The Cronbach $\alpha$ for this seven-item scale, used to test Hypothesis 1 , is 0.83 for our sample.

The second dependent variable is Task Interdependence, which is used to test Hypothesis 2. Following Kiggundu (1983), five items in the questionnaire address this construct. Following reliability analysis, three of the items were eliminated. A higher score on this scale $(\alpha=0.64)$ corresponds to the perception of a higher degree of collaboration and dependency among team members in the process of task completion.

Hypothesis 3 is tested using the third dependent variable, Information Sharing, which is aimed at capturing the degree to which respondents perceive a high degree of communication within the team. Following Bunderson and Sutcliffe (2002), three items were considered for this variable. Two ('Information used to make key decisions was freely shared in the team' and 'Members of my team freely provided their personal expertise and insight') were retained, following reliability analysis ( $\alpha$ $=0.72$ ), and higher scores suggest higher perceived levels of communication.

Two scales are employed to assess conflict within teams, both based on Jehn (1995). The first, Relationship Conflict, consists of four items that aim to measure the extent of interpersonal conflict or friction among team members, based on statements including 'There was a lot of friction among members in my team' and 'There were a lot of personality conflicts in my team'. This variable is used to test Hypothesis 4, with a higher score suggesting a higher level of interpersonal conflict, as perceived by the respondent. The Cronbach $\alpha$ of this scale is 0.78 . The second conflict-related dependent variable is Task Conflict, used to test Hypothesis 5. This four-item scale has a Cronbach $\alpha$ of 0.82 , and is also computed such that higher scores represent higher perceived levels of conflict.

Independent variable. The primary explanatory variable for this study is the Collectivism Scale of Yamaguchi (1994). This measure of collectivism is specifically intended to assess collectivism at the personal level. The scale has 10 items pertaining to respondent views regarding group membership and teamwork, measuring the extent of the respondent's agreement to statements on a seven-point Likert-type scale, where $1=$ strongly disagree and $7=$ strongly agree. This scale treats individualism and collectivism as endpoints on a continuum, such that a higher score indicates a stronger 
collectivistic orientation, while a lower score suggests a weaker collectivistic/more individualistic orientation. Yamaguchi (1994) reported reliabilities for this scale of between 0.77 and 0.88 in different samples; our sample yields a Cronbach $\alpha$ of 0.67 .

Recognizing the potential for social desirability effects to result in collectivists' responding more favorably about team processes than individualists, we undertook a comparison of means, using ANOVA. Our analysis did not reveal consistent patterns between any of our dependent variables and the personal collectivism variable, discretizing collectivism using both the $25 \mathrm{th} / 75$ th and the $33.3 \mathrm{rd} / 66.7$ th percentiles. While this does not completely negate the potential for social desirability bias, the fact that the personal-level collectivism measure, itself, does not vary systematically with the dependent variables suggests that findings pertaining to marginal relationships between this measure and the dependent variables, after controlling for other variables, are of interest.

Control variables. It is certainly reasonable to expect that assessments of team performance will be associated with other factors, in addition to the respondent's personal I/C orientation. In the context of global virtual teams, members' perceptions regarding their overall experience might be related to their fluency in the common language used for the project and their prior exposure to other cultures. We control for these attributes using two dummy variables; Native English assumes the value 1 for respondents whose native language is English, and 0 otherwise, while Lived Overseas is equal to 1 if the respondents report having lived outside their home country for at least one year (and 0 otherwise). As demographics have been shown to influence members' perceptions of teamwork in both face-to-face and virtual teams (Baugh and Graen, 1997; Jehn et al., 1999; Krebs et al., 2006), other respondent information is included in the models: Level of education (a sixlevel ordinal variable representing the highest level of education attained), Age (in years), and Gender $(1=$ female, $0=$ male $)$.

Responses are also likely to be affected by the actual team dynamics experienced during the project; these are specific to each team. Thus, we also control for attributes of the respondents' teams. For example, the size of the team has been shown to influence effectiveness, with larger teams tending to experience less interaction among team members and lower quality of communication (Hare, 1981; cited in Kirkman and Shapiro, 1997); we include the Team Size variable to control for this effect. Under the assumption that more geographically dispersed teams present more challenges, due to issues such as time zones and varying levels of infrastructure to support electronic communication, we also control for the number of countries represented on respondents' teams (Countries). To incorporate a consideration of team-specific cultural differences, we include the median individualism level (Hofstede, 2001) for each team, based on members' reported nationalities (Team I/C). In addition, we account for the culture-level I/C nature of the respondent's primary identification, based on Hofstede's $(1984,2001)$ Individualism dimension, in order to tease out subtleties associated with the individual-level relationships of interest in this study.

Table 2 shows descriptive statistics for the sample data, and Table 3 provides a correlation matrix for the continuous variables used in the study.

\section{Results}

We employ Ordinary Least Squares (OLS) regression to test our hypotheses. In order to develop a more nuanced understanding of the relationships, we present four models for each of the dependent variables. The first is a base model, while the second includes the culture-level Individualism variable. The third model controls for the team I/C level, and the fourth includes all of the variables; the results 
Table 2. Descriptive statistics

\begin{tabular}{lccccccc}
\hline & $\boldsymbol{n}$ & Mean & Median & Mode & SD & Min & Max \\
\hline Trust & 105 & 4.84 & 4.86 & & 1.18 & 1.00 & 7.00 \\
Task Interdependence & 105 & 4.80 & 5.00 & & 1.38 & 1.00 & 6.40 \\
Information Sharing & 105 & 5.07 & 5.00 & & 1.26 & 1.00 & 7.00 \\
Relationship Conflict & 104 & 2.74 & 2.50 & & 1.22 & 1.00 & 6.50 \\
Task Conflict & 104 & 3.11 & 3.00 & & 1.28 & 1.00 & 6.75 \\
Collectivism Scale & 105 & 4.99 & 5.00 & & 0.69 & 3.20 & 6.70 \\
Native English?* & 105 & & 0.00 & 0 & & 0 & 1 \\
Lived Overseas?* & 105 & & 1.00 & 1 & & 0 & 1 \\
Education Level** & 104 & & 3.00 & 3 & & 1 & 5 \\
Age & 104 & 22.96 & 22.00 & & 3.70 & 18 & 44 \\
Gender*** & 105 & & 1.00 & 1 & & 0 & 1 \\
Team Size & 105 & 6.43 & 7.00 & & 1.54 & 1 & 8 \\
Countries on Team & 105 & 3.87 & 4.00 & & 0.84 & 1 & 6 \\
Individualism & 105 & 47.64 & 48.00 & & 25.71 & 18.00 & 94.00 \\
Team I/C & 105 & 50.60 & 49.50 & & 13.23 & 20.00 & 79.50 \\
\hline
\end{tabular}

* Values for Native English? and Lived Overseas? Are: I=Yes, $0=$ No

** Values for Education Level are: I=Some secondary, 2=Completed secondary, 3=Some university, 4=Completed undergraduate, $5=$ Completed graduate, $6=$ Completed $\mathrm{PhD} /$ doctorate

*** Values for Gender are: I=Female, $0=$ Male

Table 3. Correlation matrix

\begin{tabular}{lcccccccccc}
\hline & 1 & 2 & 3 & 4 & 5 & 6 & 7 & 8 & 9 & 10 \\
\hline I.Trust & & & & & & & & & & \\
2.Task Interdependence & $.64^{* *}$ & & & & & & & & & \\
3. Information Sharing & $.77^{* *}$ & $.55^{* *}$ & & & & & & & & \\
4. Relationship Conflict & $.47^{* *}$ & .19 & $.45^{* *}$ & & & & & & & \\
5. Task Conflict & $.38^{* *}$ & $.21^{*}$ & $.42^{* *}$ & $.74^{* *}$ & & & & & & \\
6. Collectivism Scale & $.23^{*}$ & $.31^{* *}$ & $.26^{* *}$ & .10 & .13 & & & & & \\
7. Age & .04 & $.36^{*}$ & .02 & .18 & .05 & $.33^{* *}$ & & & & \\
8. Team Size & .11 & .09 & .00 & .09 & .01 & .00 & .16 & & & \\
9. Countries on Team & .13 & .10 & .03 & $.20^{*}$ & $.28^{* *}$ & .05 & .09 & $.38^{* *}$ & & \\
I0. Individualism & $.31^{* *}$ & $.41^{* *}$ & $.25^{*}$ & .05 & .01 & $.21^{*}$ & .11 & .12 & .08 & \\
II. Team I/C & .02 & .11 & .18 & .03 & .04 & .11 & .08 & .10 & $.33^{* *}$ & .06 \\
\hline
\end{tabular}

\footnotetext{
$* * p<0.01$

$*_{p}<0.05$

two-tailed tests

$n=104$ or 105
}

are shown in Tables 4-8. The various models provide very consistent decisions for each of the dependent variables, which is not surprising, given that the Individualism and Team I/C variables contribute minimal marginal explanatory power. Despite some pairwise correlations that differ significantly from 
Table 4. Regression results for Trust (unstandardized coefficients, standard errors in parentheses)

\begin{tabular}{lcccc}
\hline & Base Model & $\begin{array}{c}\text { With Culture-Level } \\
\text { Individualism }\end{array}$ & $\begin{array}{c}\text { With Median } \\
\text { Team I/C }\end{array}$ & Full Model \\
\hline Constant & $2.36^{\dagger}(1.22)$ & $2.85^{*}(1.26)$ & $2.01(1.32)$ & $2.44^{\dagger}(1.35)$ \\
Collectivism Scale & $0.44^{*}(0.18)$ & $0.40^{*}(0.18)$ & $0.43^{*}(0.18)$ & $0.38^{*}(0.18)$ \\
Native English? & $0.83^{* *}(0.29)$ & $0.57(0.34)$ & $0.85^{* *}(0.29)$ & $0.57^{\dagger}(0.34)$ \\
Lived Overseas? & $0.00(0.24)$ & $0.06(0.25)$ & $0.10(0.24)$ & $0.08(0.25)$ \\
Education Level & $0.19(0.16)$ & $0.17(0.16)$ & $0.20(0.16)$ & $0.18(0.16)$ \\
Age & $0.06(0.04)$ & $0.05(0.04)$ & $0.06(0.04)$ & $0.06(0.04)$ \\
Gender & $0.10(0.22)$ & $0.10(0.22)$ & $0.09(0.23)$ & $0.08(0.22)$ \\
Team Size & $0.02(0.08)$ & $0.00(0.08)$ & $0.02(0.08)$ & $0.00(0.08)$ \\
Countries on Team & $0.27^{\dagger}(0.15)$ & $0.30^{*}(0.15)$ & $0.30^{\dagger}(0.16)$ & $0.34^{*}(0.16)$ \\
Individualism & & $0.01(0.01)$ & & $0.01(0.01)$ \\
Team I/C & & & $0.01(0.01)$ & $0.01(0.01)$ \\
$n$ & 103 & 103 & 103 & 103 \\
$R^{2}$ & 0.18 & 0.20 & 0.19 & 0.21 \\
$R^{2}{ }_{\text {adj }}$ & 0.12 & 0.12 & 0.11 & 0.12 \\
Max VIF & 1.48 & 1.77 & 1.50 & 1.79 \\
\hline
\end{tabular}

$* * p<0.01$

$*_{p}<0.05$

${ }^{\dagger} p<0.10$

two-tailed tests

zero, none of the regression models suffers from problem multicollinearity, as demonstrated by variance inflation factors (VIFs) all below 2.0. Residual analysis suggests that the OLS assumptions are met reasonably well, with no evidence of heteroscedasticity in any of the models.

The results in Table 4 pertain to the relationship between collectivism and trust, in the context of these multicultural global virtual teams. The estimated coefficients associated with the Collectivism Scale variable are positive and significant $(p<0.05)$ for all four of the models, providing support for Hypothesis 1. In addition, native English speakers report significantly lower levels of trust, holding all of the other variables constant in three of the four models, and a larger number of countries represented within the team is associated with higher levels of trust in all of the models.

Similarly, Hypothesis 2 is supported, based on the positive and significant $(p<0.05)$ estimated coefficients associated with the Collectivism Scale variable in the four models of Task Interdependence, shown in Table 5. Older respondents also report significantly more favorable perceptions of task interdependence in their global virtual teams, across all four models. In addition, respondents whose native language is English report lower $(p<0.01)$ levels of perceived task interdependence in their teams, marginal to the other variables, when Individualism is not included as a control variable. The coefficient associated with the culture-level Individualism variable is negative when Team I/C is not included in the model, and positive in the full model. While the mean levels of Individualism differ significantly between native and non-native English speakers, based on $t$-tests, the low VIFs show that mutlicollinearity is not affecting the estimation and interpretation of the estimated coefficients in the regression models.

Hypothesis 3 also receives strong support from the data, with positive relationships between Collectivism Scale and Information Sharing (at least $p<0.05$ ); see Table 6. Among the control 
Table 5. Regression results for Task Interdependence (unstandardized coefficients, standard errors in parentheses)

\begin{tabular}{lcccr}
\hline & Base Model & $\begin{array}{c}\text { With Culture-Level } \\
\text { Individualism }\end{array}$ & $\begin{array}{c}\text { With Median } \\
\text { Team I/C }\end{array}$ & Full Model \\
\hline Constant & $0.22(1.32)$ & $0.54(1.36)$ & $0.63(1.42)$ & $1.02(1.44)$ \\
Collectivism Scale & $0.44^{*}(0.19)$ & $0.38^{*}(0.19)$ & $0.46^{*}(0.19)$ & $0.40^{*}(0.19)$ \\
Native English? & $1.00^{* *}(0.31)$ & $0.59(0.37)$ & $0.95^{* *}(0.31)$ & $0.58(0.37)$ \\
Lived Overseas? & $0.20(0.26)$ & $0.10(0.26)$ & $0.23(0.26)$ & $0.13(0.26)$ \\
Education Level & $0.02(0.17)$ & $0.02(0.17)$ & $0.01(0.17)$ & $0.04(0.17)$ \\
Age & $0.08^{\dagger}(0.04)$ & $0.09^{*}(0.04)$ & $0.09 *(0.04)$ & $0.09^{*}(0.04)$ \\
Gender & $0.24(0.24)$ & $0.24(0.24)$ & $0.27(0.24)$ & $0.26(0.24)$ \\
Team Size & $0.08(0.09)$ & $0.06(0.09)$ & $0.08(0.09)$ & $0.06(0.09)$ \\
Countries on Team & $0.11(0.16)$ & $0.15(0.16)$ & $0.03(0.17)$ & $0.08(0.17)$ \\
Individualism & & $0.01^{\dagger}(0.01)$ & & $0.01^{\dagger}(0.01)$ \\
Team I/C & & & $0.02(0.01)$ & $0.01(0.01)$ \\
$n$ & 103 & 103 & 103 & 103 \\
$R^{2}$ & 0.31 & 0.33 & 0.32 & 0.35 \\
$R_{\text {adj }}^{2}$ & 0.25 & 0.27 & 0.26 & 0.28 \\
Max VIF & 1.48 & 1.77 & 1.50 & 1.79 \\
\hline
\end{tabular}

$* * p<0.01$

$*_{p}<0.05$

$t_{p}<0.10$

two-tailed tests

variables, younger respondents tend to perceive less extensive sharing of information within the virtual teams, after controlling for the team's median I/C level, as do native English speakers in one of the models. In addition, a higher median I/C level for the team is associated with more positive views about information sharing.

Hypotheses 4 and 5 posit that collectivists will, on average, perceive less conflict in the team environment, compared to individualists. We find limited support for Hypothesis 4, which considers Relationship Conflict, as only two of the models provide evidence of a significant $(p<0.10)$ negative relationship between a person's collectivism level and his or her perception of relational conflict in the team, after accounting for the control variables; see Table 7. In addition, older respondents tend to perceive more relationship conflict, while teams with representatives of more countries appear to generate lower levels of conflict. Hypothesis 5 receives stronger support, with negative relationships between Collectivism Scale and Task Conflict $(p<0.05)$ in all four models, and similar results with respect to the number of countries represented in the team and the perceived level of conflict.

\section{Conclusion}

As multinational enterprises seek to compete more effectively, many are moving toward developing flatter, more flexible organizational structures, to facilitate greater productivity. The use of short-duration, project-oriented teams is complementary to these goals. Recent improvements in information and communication technologies mean that such working groups can span boundaries, permitting the creation of short-term virtual teams. Whether these transient, long-distance teams 
Table 6. Regression results for Information Sharing (unstandardized coefficients, standard errors in parentheses)

\begin{tabular}{lcccc}
\hline & Base Model & $\begin{array}{c}\text { With Culture-Level } \\
\text { Individualism }\end{array}$ & $\begin{array}{c}\text { With Median } \\
\text { Team I/C }\end{array}$ & Full Model \\
\hline Constant & $3.08^{*}(1.39)$ & $3.50^{*}(1.44)$ & $2.23(1.46)$ & $2.66^{\dagger}(1.49)$ \\
Collectivism Scale & $0.57^{* *}(0.19)$ & $0.53^{* *}(0.19)$ & $0.54^{* *}(0.19)$ & $0.50^{*}(0.19)$ \\
Native English? & $0.50(0.32)$ & $0.26(0.38)$ & $0.53^{\dagger}(0.32)$ & $0.25(0.37)$ \\
Lived Overseas? & $0.39(0.27)$ & $0.33(0.27)$ & $0.38(0.27)$ & $0.31(0.27)$ \\
Education Level & $0.04(0.17)$ & $0.02(0.17)$ & $0.07(0.17)$ & $0.05(0.17)$ \\
Age & $0.06(0.04)$ & $0.06(0.04)$ & $0.07^{\dagger \cdot}(0.04)$ & $0.07^{\dagger}(0.04)$ \\
Gender & $0.07(0.25)$ & $0.07(0.25)$ & $0.11(0.24)$ & $0.11(0.24)$ \\
Team Size & $0.03(0.09)$ & $0.04(0.09)$ & $0.04(0.09)$ & $0.06(0.09)$ \\
Countries on Team & $0.17(0.17)$ & $0.19(0.17)$ & $0.25(0.17)$ & $0.28(0.17)$ \\
Individualism & & $0.01(0.01)$ & & $0.01(0.01)$ \\
Team I/C & & 102 & $0.02^{\dagger}(0.01)$ & $0.02^{\dagger}(0.01)$ \\
$n$ & 102 & 0.16 & 102 & 102 \\
$R^{2}$ & 0.15 & 0.08 & 0.18 & 0.20 \\
$R_{\text {adj }}^{2}$ & 0.08 & 1.75 & 0.10 & 0.11 \\
Max VIF & 1.47 & & 1.50 & 1.77 \\
\hline
\end{tabular}

$* * p<0.01$

$*_{p}<0.05$

$+p<0.10$

two-tailed tests

include participants from various international subsidiaries or draw their members from increasingly multicultural domestic workforces, the management of cultural differences is expected to be more complex than in environments characterized by face-to-face communications. The goal of this research is to understand more about how culture is related to group dynamics, in the context of global virtual teams.

Based on a survey of participants in short-duration, project-oriented global virtual teams, we find evidence that respondents with stronger collectivist orientations tend to report more favorable impressions of team processes, compared with individualists. These results are consistent with respect to trust, task interdependence, information sharing, and task conflict, both with and without controlling for the respondents' culture-level I/C orientations based on Hofstede's individualism dimension; only the perception of relationship conflict within the teams does not demonstrate a marginal relationship with personal collectivism. Our finding that collectivists tend to display prototypical attitudes about teamwork, even in the context of an ad hoc multicultural virtual team setting, provides strong - and perhaps surprising - evidence about the strength of cultural orientation. Communication via virtual means does not mitigate cultural differences, despite the resultant inability to foster interpersonal face-to-face relationships.

This study is, of course, subject to limitations. The members of the global virtual teams in our study were primarily students, and their perceptions may differ somewhat from those of participants in more typical business environments. In addition, we have measured post hoc perceptions rather than real-time ones, although responses were collected immediately upon the completion of the team assignments, while the global virtual team experience was still fresh in the minds of 
Table 7. Regression results for Relationship Conflict (unstandardized coefficients, standard errors in parentheses)

\begin{tabular}{lcccr}
\hline & Base Model & $\begin{array}{c}\text { With Culture-Level } \\
\text { Individualism }\end{array}$ & $\begin{array}{c}\text { With Median } \\
\text { Team I/C }\end{array}$ & \multicolumn{1}{c}{ Full Model } \\
\hline Constant & $3.94^{* *}(1.37)$ & $3.60^{*}(1.42)$ & $4.0 I^{* *}(1.46)$ & $3.72^{*}(1.50)$ \\
Collectivism Scale & $0.32^{\dagger}(0.19)$ & $0.29(0.19)$ & $0.32^{\dagger}(0.19)$ & $0.29(0.19)$ \\
Native English? & $0.02(0.31)$ & $0.16(0.37)$ & $0.02(0.32)$ & $0.17(0.37)$ \\
Lived Overseas? & $0.07(0.26)$ & $0.12(0.27)$ & $0.07(0.27)$ & $0.12(0.27)$ \\
Education Level & $0.16(0.16)$ & $0.14(0.17)$ & $0.16(0.17)$ & $0.14(0.17)$ \\
Age & $0.10^{*}(0.04)$ & $0.09^{*}(0.04)$ & $0.10^{*}(0.04)$ & $0.10^{*}(0.04)$ \\
Gender & $0.06(0.24)$ & $0.06(0.24)$ & $0.06(0.24)$ & $0.06(0.24)$ \\
Team Size & $0.03(0.09)$ & $0.04(0.09)$ & $0.04(0.09)$ & $0.05(0.09)$ \\
Countries on Team & $0.44^{* *}(0.16)$ & $0.46^{* *}(0.16)$ & $0.45^{* *}(0.17)$ & $0.47^{* *}(0.17)$ \\
Individualism & & $0.01(0.01)$ & & $0.00(0.01)$ \\
Team I/C & & & $0.00(0.01)$ & $0.01(0.01)$ \\
$n$ & 102 & 102 & 102 & 102 \\
$R^{2}$ & 0.13 & 0.14 & 0.13 & 0.14 \\
$R_{\text {adj }}^{2}$ & 0.05 & 0.05 & 0.04 & 0.04 \\
Max VIF & 1.47 & 1.75 & 1.50 & 1.77 \\
\hline
\end{tabular}

$* * p<0.01$

$*_{p}<0.05$

${ }^{+} p<0.10$

two-tailed tests

participants. The fact that the survey was conducted in English must also be acknowledged; when responding in a non-native language, participants may accommodate some of their responses to reflect their perceptions of the culture represented by the language of the survey instrument (Harzing et al., 2005). However, the working language of the teams was English, which is expected to mitigate this effect.

The size and composition of our sample limited our options with respect to assessing team-level perceptions. Kirkman and Shapiro (2005) emphasized the value of measuring mean levels of cultural values and cultural diversity in multicultural teams. While the fact that the respondents in our study were spread across a wide number of teams enhances the sample's representativeness, it also precluded our incorporation of team-level measures into the models. Including more detailed information regarding members' cultural values and the mutual distances within each global virtual team would enhance the analysis.

In this study, we have focused on a single cultural dimension. While we have measured I/C at both the group and personal levels, we recognize that individualism and collectivism do not operate in isolation. Studies on team perceptions and processes would benefit from an examination of the interactions among different values dimensions at both levels of analysis. While we find no evidence that our findings are driven by social desirability effects, additional investigation into this issue would also advance our understanding.

Our study has demonstrated that cultural values are related to perceived levels of conflict, trust, and interdependence in global virtual teams. A comparison of these relationships between virtual and face-to-face team situations is an important area for future inquiry. Recent research has suggested that relationship conflict, which is associated with lower levels of trust, 
Table 8. Regression results for Task Conflict (unstandardized coefficients, standard errors in parentheses)

\begin{tabular}{lrrrr}
\hline & Base Model & $\begin{array}{c}\text { With Culture-Level } \\
\text { Individualism }\end{array}$ & $\begin{array}{c}\text { With Median } \\
\text { Team I/C }\end{array}$ & \multicolumn{1}{c}{ Full Model } \\
\hline Constant & $6.85^{* *}(\mathrm{I} .34)$ & $6.75^{* *}(\mathrm{I} .40)$ & $7.18^{* *}(\mathrm{I} .43)$ & $7.07^{* *}(\mathrm{I} .47)$ \\
Collectivism Scale & $0.4 \mathrm{I}^{*}(0.18)$ & $0.40^{*}(0.19)$ & $0.40^{*}(0.18)$ & $0.39^{*}(0.19)$ \\
Native English? & $0.04(0.3 \mathrm{I})$ & $0.10(0.37)$ & $0.03(0.3 \mathrm{I})$ & $0.10(0.37)$ \\
Lived Overseas? & $0.10(0.26)$ & $0.09(0.26)$ & $0.10(0.26)$ & $0.08(0.27)$ \\
Education Level & $0.16(0.16)$ & $0.16(0.16)$ & $0.18(0.16)$ & $0.17(0.16)$ \\
Age & $0.04(0.04)$ & $0.04(0.04)$ & $0.05(0.04)$ & $0.04(0.04)$ \\
Gender & $0.08(0.24)$ & $0.08(0.24)$ & $0.07(0.24)$ & $0.07(0.24)$ \\
Team Size & $0.07(0.09)$ & $0.07(0.09)$ & $0.07(0.09)$ & $0.07(0.09)$ \\
Countries on Team & $0.65^{* *}(0.16)$ & $0.65^{* *}(0.16)$ & $0.68 * *(0.17)$ & $0.69 * *(0.17)$ \\
Individualism & & $0.00(0.01)$ & & $0.00(0.01)$ \\
Team I/C & & & $0.01(0.01)$ & $0.01(0.01)$ \\
$n$ & 102 & 102 & 102 & 102 \\
$R^{2}$ & 0.19 & 0.19 & 0.19 & 0.19 \\
$R^{2}{ }_{\text {adj }}$ & 0.12 & 0.11 & 0.11 & 0.10 \\
Max VIF & 1.47 & 1.75 & 1.50 & 1.77 \\
\hline
\end{tabular}

$* * p<0.01$

$*_{p}<0.05$

${ }^{\dagger} p<0.10$

two-tailed tests

negatively influences task interdependence in self-managed teams (Langfred, 2007). Thus, there is a clear need for further research that unifies existing findings and expands models of team performance, by including moderating effects of interpersonal interaction both in distributed and co-located team settings.

The brevity of the project considered in this study should also be noted. Over time, members of multicultural teams may develop a strong cohesion that mitigates the importance of their cultural differences (Randel, 2003). The three-week duration of the virtual teams in this study did not offer participants - especially those with strongly collectivist orientations - very much time to form relationships and develop in-group cohesion. Yet, the fact that we observe significant and consistent relationships between collectivism orientation and the perception of team processes, despite the limited duration, suggests that cultural differences remain strong, despite the virtual nature of the context.

This research builds on studies that have focused on teamwork and cross-cultural communication via electronic networks (e.g., Jarvenpaa and Leidner, 1999), in an effort to contribute to an understanding of the complex interactions that characterize global virtual teams and provide insights into the impact of the cultural composition of team members and their potentially diverging perceptions of team processes. Our results suggest that, although the development of relationships is constrained by the short-term, transient nature of such groups and the likely lack of familiarity among the personnel, team members with collectivist personal orientations may still perceive the team-related processes and interactions more favorably than their more individualist counterparts. This finding is somewhat counter-intuitive, as one would expect team members with individualist orientations and strong task-focus to perform better in global virtual teams and to evaluate such work more favorably than their relationship-focused, collectivist counterparts. 
Given these findings, it may be time to rethink the role of the global virtual team, with respect to building on its potential for organizing people and projects, with the goal of enhancing competitive advantage. While individualists within such teams are more task-focused and may be motivated by personal rewards, collectivists seem able to form rapid loyalty to, and trust in, the group to which they have been assigned. Understanding these distinctions should enhance managerial effectiveness with respect to both organizations and teams. For example, with proper nurturing, collectivists' loyalty and trust may be transferred to the institution or to the group as an organizational form, with the potential to act as 'quick glue' that will assist in the development of group norms and a shared context. Subsequent research may help to refine such notions, and allow more tailored facilitation of the contributions of individualists and collectivists toward improving performance and creating a shared social context.

\section{Acknowledgements}

We are especially grateful to Richard Brislin and an anonymous reviewer for their insightful comments on earlier versions of this manuscript.

\section{References}

Baugh SG and Graen GB (1997) Effects of team gender and racial composition on perceptions of team performance in cross-functional teams, Group and Organization Management 22: 366-83.

Bierbrauer G, Meyer H, and Wolfradt U (1994) Measurement of normative and evaluative aspects of individualistic and collectivistic orientations: the cultural orientation scale, in Kim et al., Individualism and Collectivism: Theory, Method and Applications. Thousand Oaks, CA: SAGE, 189-99.

Bunderson JS and Sutcliffe KM (2002) Comparing alternative conceptualizations of functional diversity in management teams: process and performance effects. Academy of Management Journal 45: 875-93.

Cox T, Lobel S, and McLeod P (1991) Effects of ethnic group cultural differences on cooperative and competitive behavior on a group task. Academy of Management Journal 34: 827-47.

Earley PC (1989) Social loafing and collectivism: a comparison of the United States and the People's Republic of China. Administrative Science Quarterly 34(4): 565-81.

Erdem F and Ozen J (2000) Trust and distrust in teamwork: unconditional trust or optimal trust for performance? Paper presented at the 8th National Management and Organizational Congress, Nevsehir, Turkey.

Fukuyama F (1995) Trust: The Social Virtues and the Creation of Prosperity. New York: Free Press.

Gomez C, Kirkman BL, and Shapiro DJ (2000) The impact of collectivism and in-group/out-group membership on the evaluation generosity of team members. Academy of Management Journal 43(6): 1097-1106.

Handy C (1995) Trust and the virtual organization: how do you manage people who you do not see? Harvard Business Review 73(3): 40-50.

Hare AP (1981) Group size. American Behavioral Scientist 24: 695-708.

Harzing AW et al (2005) The use of English questionnaires in cross-national research: does cultural accommodation obscure national differences? International Journal of Cross-Cultural Management 5(2): 213-24.

Hofstede G (1984) Culture's Consequences: International Differences in Work-Related Values. Thousand Oaks, CA: SAGE.

Hofstede G (2001) Culture's Consequences: Comparing Values, Behaviors, Institutions, and Organizations across Nations. Thousand Oaks, CA: SAGE.

Jarvenpaa S and Leidner DE (1999) Communication and trust in global virtual teams. Organization Science 10 (6): 791-815.

Jehn KA (1995) A multimethod examination of the benefits and detriments of intragroup conflict. Administrative Science Quarterly 40(2): 256-82.

Jehn KA, Northcraft GB, and Neale MA (1999) Why differences make a difference: a field study of diversity, conflict, and performance in workgroups. Administrative Science Quarterly 44: 741-63. 
Kagitcibasi C (1994) A critical appraisal of individualism and collectivism: toward a new formulation. In: Kim,? et al. (eds) Individualism and Collectivism: Theory, Method and Applications. Thousand Oaks, CA: SAGE, 52-65.

Kiffin-Petersen SA and Cordery JL (2003) Trust, individualism and job characteristics as predictors of employee preference for teamwork. International Journal of Human Resource Management 14(1): 93-116.

Kiggundu MN (1983) Task interdependence and job design: test of a theory. Organizational Behavior and Human Decision Processes 31: 145-72.

Kim KI, Park H, and Suzuki N (1990) Reward allocations in the United States, Japan and Korea: a comparison of individualistic and collectivistic cultures. Academy of Management Journal 33: 188-98.

Kirkman BL and Shapiro DL (1997) The impact of cultural values on employee resistance to teams: toward a model of globalized self-managing work team effectiveness. Academy of Management Review 22(3): $730-57$.

Kirkman BL and Shapiro DL (2001) The impact of cultural values on job satisfaction and organizational commitment in self-managing work teams: the mediating role of employee resistance. Academy of Management Journal, 44: 557-69.

Kirkman BL, Lowe KB, and Gibson CB (2006). A quarter century of Culture's Consequences: a review of empirical research incorporating Hofstede's cultural values framework. Journal of International Business Studies 37: 285-320.

Kogut B and Zander U (1995) Knowledge and the speed of the transfer and imitation of organizational capabilities. Organizational Science 6: 76-92.

Kramer RM (1994) The sinister attribution error: paranoid cognition and collective distrust in organizations. Motivation and Emotion 18: 199-230.

Krebs SA, Hobman EV, and Bordia P (2006) Virtual teams and group member dissimilarity: consequences for the development of trust. Small Group Research 37: 721-41.

Kristof AL, Brown KG, Sims HP, and Smith KA (1995) The virtual team: a case study and inductive model. In: Beyerlein? et al. (eds) Advances in Interdisciplinary Studies of Work Teams: Knowledge Work in Teams. Greenwich: JAI Press, vol. 2, 229-53.

Langfred CW (2007) The downside of self-management: a longitudinal study of the effects of conflict on trust, autonomy, and task-interdependence in self-managing teams. Academy of Management Journal 50(4): 885-900.

Lewicki RJ, Tomlinson EC, and Gillespie N (2006) Models of interpersonal trust development: theoretical approaches, empirical evidence, and future directions. Journal of Management 32: 991-1022.

Matveev AV and Nelson PE (2004) Cross cultural communication competence and multicultural team performance: perceptions of American and Russian managers. International Journal of Cross Cultural Management 4(2): 253-70.

Mayer RC, Davis JH, and Schooman FD (1995) An integrative model of organizational trust. Academy of Management Review 20: 709-34.

Maznevski M, DiStefano JJ, Gomez CB, Noorderhaven NG, and Wu PC (2002) Cultural dimensions at the individual level of analysis. International Journal of Cross-Cultural Management 2(3): 275-95.

Mowshowitz A (1997) Virtual organization. Communications of the ACM 40(9): 30-7.

Nohria N and Eccles RG (1992) Face-to-face: making network organizations work. In: Nohria N and Eccles RG, Network and Organization. Boston, MA: Harvard Business School Press, 288-308.

O'Hara-Devereaux M and Johansen R (1994) Global Work: Bridging Distance. San Francisco, CA: JosseyBass.

Palich LE, Hom PW, and Griffeth RW (1995) Managing in the international context: testing the cultural generality of sources of commitment to a multinational enterprise. Journal of Management 21: 671-90.

Randel AE (2003) The salience of culture in multinational teams and its relation to team citizenship behavior. International Journal of Cross Cultural Management 3(1): 27-44.

Sosik JJ and Jung DI (2002) Work-group characteristics and performance in collectivistic and individualistic cultures. Journal of Social Psychology 142(1): 5-23. 
Triandis HC (1994) Theoretical and methodological approaches to the study of collectivism and individualism. In: Kim? et al. (eds) Individualism and Collectivism: Theory, Method and Applications. Thousand Oaks, CA: SAGE, 41-51.

Triandis HC (1995) Individualism and Collectivism. Oxford: Westview Press.

Triandis HC (2004) The many dimensions of culture. Academy of Management Executive 18(1): 88-93.

Triandis HC, Leung K, Villareal MV, and Clark FL (1985) Allocentric versus idiocentric tendencies: convergent and discriminant validation. Journal of Research in Personality 19: 395-415.

Wagner JA (1995) Studies of individualism-collectivism: effects on cooperation in groups. Academy of Management Journal 38(1): 152-72.

Yamaguchi S (1994) Collectivism among the Japanese: a perspective from the self. In: Kim? et al. (eds) Individualism and Collectivism: Theory, Method and Applications. Thousand Oaks, CA: SAGE, 157-74. 